\section{RMD Open}

Rheumatic \&

Musculoskeletal Diseases

\title{
Storm, typhoon, cyclone or hurricane in patients with COVID-19? Beware of the same storm that has a different origin
}

\author{
Alessia Alunno (iD, ${ }^{1}$ Francesco Carubbi $\mathbb{1},{ }^{2}$ Javier Rodríguez-Carrio (iD ${ }^{3}$
}

To cite: Alunno A, Carubbi F, Rodríguez-Carrio J. Storm, typhoon, cyclone or hurricane in patients with COVID-19? Beware of the same storm that has a different origin. RMD Open 2020;6:e01295. doi:10.1136/ rmdopen-2020-001295

Received 29 April 2020 Revised 5 May 2020 Accepted 7 May 2020
D Check for updates

(C) Author(s) (or their employer(s)) 2020. Re-use permitted under CC BY-NC. No commercial re-use. See rights and permissions. Published by BMJ.

${ }^{1}$ Department of Medicine, Rheumatology Unit, University of Perugia, Perugia, Italy

${ }^{2}$ Department of Biotechnological and Applied Clinical Sciences, Rheumatology Unit, University of L'Aquila, L'Aquila, Italy ${ }^{3}$ University of Oviedo, Instituto de Investigación Sanitaria del Principado de Asturias, Oviedo, Spain

Correspondence to Alessia Alunno; alessia.alunno82@gmail.com

\section{ABSTRACT}

Some of the articles being published during the severe acute respiratory syndrome-coronavirus (SARS-CoV)-2 pandemic highlight a link between severe forms of coronavirus disease 2019 (COVID-19) and the so-called cytokine storm, also with increased ferritin levels. However, this scenario is more complex than initially thought due to the heterogeneity of hyperinflammation. Some patients with coronavirus 2019 disease (COVID-19) develop a fully blown secondary haemophagocytic lymphohistiocytosis (sHLH), whereas others, despite a consistent release of pro-inflammatory cytokines, do not fulfil sHLH criteria but still show some features resembling the phenotype of the hyperferritinemic syndrome. Despite the final event (the cytokine storm) is shared by various conditions leading to $\mathrm{SHLH}$, the aetiology, either infectious, autoimmune or neoplastic, accounts for the differences in the various phases of this process. Moreover, the evidence of a hyperinflammatory microenvironment provided the rationale to employ immunomodulating agents for therapeutic purposes in severe COVID-19. This viewpoint aims at discussing the pitfalls and issues to be considered with regard to the use of immunomodulating agents in COVID-19, such as timing of treatment based on the viral load and the extent of cytokine/ ferritin overexpression. Furthermore, it encompasses recent findings in the paediatric field about a novel multisystem inflammatory disease resembling toxic shock syndrome and atypical Kawasaki disease observed in children with proven SARS-CoV2 infection. Finally, it includes arguments in favour of adding COVID-19 to the spectrum of the recently defined 'hyperferritinemic syndrome', which already includes adult-onset Still's disease, macrophage activation syndrome, septic shock and catastrophic anti-phospholipid syndrome.

\section{BACKGROUND}

Since December 2019, the scientific community all over the globe has been confronted with an unprecedented challenge, a battle against a new infectious agent, the severe acute respiratory syndrome-coronavirus (SARS-CoV)-2, subsequently defined a pandemic by the WHO, with a worrisomely high mortality rate. Although in the majority of patients the coronavirus 2019 disease (COVID-19) causes only mild-to-moderate symptoms, a consistent proportion of infected subjects could develop respiratory failure, acute respiratory distress syndrome (ARDS) and sepsis. ${ }^{1}$ From an immunological perspective, the severity of COVID-19 seems to correlate with an increased amount of several cytokines, in particular interleukin (IL)-1 $\beta$, IL-2, IL-6, IL-7, IL-10, tumour necrosis factor- $\alpha$ (TNF $\alpha)$, granulocyte colony-stimulating factor (G-CSF), interferon (IFN) $\gamma$-induced protein $10 \mathrm{kDa} /$ CXCL10, monocyte chemoattractant protein 1 , macrophage inflammatory protein $1-\alpha$ both in serum and affected tissues. ${ }^{2} 3$ In addition, $\mathrm{CD}^{+} \mathrm{T}$ cells are activated to become pathogenic Th1 cells and generate G-CSF, thereby augmenting the expression of IL-6 in $\mathrm{CD} 14^{+} \mathrm{CD} 16^{+}$monocytes. ${ }^{4}$ Such massive release of pro-inflammatory mediators, further fuelled by positive feedback loops and aberrant activation of the immune system, resembles the socalled cytokine release syndrome, a group of conditions sharing the same pathogenic mechanism, although with a different aetiology. ${ }^{5}$ In COVID-19, this cytokine storm accounts for the two main causes of mortality in this disease: (1) ARDS and (2) secondary haemophagocytic lymphohistiocytosis (sHLH), the latter occurring in a small subset of patients. ${ }^{6}$ Needless to say how comforting it was to hypothesise a therapeutic role of immunosuppression in severe COVID-19, and over the last few weeks, increasing evidence supported that these immunomodulating compounds may be beneficial in severe COVID19. ${ }^{67}$ However, to guide therapeutic decisions, the heterogeneity under the umbrella of cytokine storm syndromes must be appraised. ${ }^{5}$

\section{COVID-19 and cytokine storms in adults}

Initially described in the early 1990 s, following the administration of the anti-T-cell antibody muromonab-CD3, the cytokine storm has been linked to different other conditions and therapeutic agents overtime, thereby broadening the spectrum of this disease. 
Nowadays, the term 'cytokine storm' applies to several fields of medicine being a potentially serious complication of immunotherapy and several infectious, neoplastic and autoimmune diseases.

Haemophagocytic lymphohistiocytosis (HLH) is a T-cell-driven hyperinflammatory, hyperferritinemic condition characterised by persistent IFN $\gamma$-dependent stimulation of Toll-like receptors (TLRs), antigenpresenting cells and T-cell uncontrolled activation, ultimately leading to a cytokine storm. ${ }^{9}$ According to the 2019 Histiocyte Society recommendations, HLH therapeutic strategies are based on the concept that it is a heterogeneous disease of variable aetiology and severity; hence, treatment should be tailored to control hyperinflammation, with glucocorticoids, intravenous immunoglobulin, cyclosporine and etoposide being the historical anchor drugs, and to treat identified disease triggers. Other immunomodulating agents like IL-1 or IL-6 inhibitors are only recommended in selected cases including the macrophage activation syndrome (MAS), a subtype of sHLH associated with systemic juvenile idiopathic arthritis (sJIA), adult-onset Still's disease (AOSD) and other autoimmune disorders. ${ }^{10}$

A context of infection by a new viral agent adds another layer of complexity to the management of the cytokine storm, thus challenging the translation of the evidence from autoimmune diseases. The causal relation between SARS-CoV2 and sHLH relies in its ability to bind TLRs and induce the above-mentioned cascade via activation of the inflammasome and release of IL-1 $3 .{ }^{11}$ Importantly, lung tissue samples collected from patients with SARSCoV during the SARS epidemic in the early 2000s have been reported to exhibit hemophagocytosis features. ${ }^{12}$ However, not all patients with COVID-19 develop a fully blown sHLH, and those with worse prognosis may neither fulfil HLH-2004 diagnostic criteria (table 1) ${ }^{13}$ nor reach the HLH-probability calculator (HScore) cut-off values. The authors who developed the HScore pointed out that as each underlying disease is associated with peculiar biologic abnormalities, the cut-off values for laboratory criteria may need to be set accordingly. ${ }^{14}$ Ruan et al described significantly higher ferritin levels in severe COVID-19, putting forward the hypothesis that it can represent a prognostic marker in this disease. However, the mean ferritin values in patients with better prognosis were still over the HLH-2004 cut-off ( $\geq 500 \mu \mathrm{g} / \mathrm{L})$, and it is unclear how many of the deceased patients reached the HScore ferritin cut-off $(2000 \mathrm{ng} / \mathrm{ml})$ and at which stage of the disease. ${ }^{15}$ Recently, it has been proposed that AOSD, MAS, catastrophic anti-phospholipid syndrome and septic shock, characterised by similar clinical picture and very elevated serum ferritin levels, are included in the so-called hyperferritinaemic syndrome. ${ }^{16}$ Interestingly, an association between pulmonary involvement and HLH was reported in patients with sJIA mediated by gene pathways related to the IFN $\gamma$ response, ${ }^{17}$ and lung imaging pattern and inflammatory mediators appear to be similar to those of patients with COVID-19. On this
Table 1 Haemophagocytic lymphohistiocytosis (HLH) 2004 diagnostic criteria $^{13}$

1. A molecular diagnosis consistent with $\mathrm{HLH}$

2. At least five of the following criteria should be met:

- Fever

Splenomegaly

- Cytopenias (affecting $\geq 2$ of 3 lineages in the peripheral blood)

- Haemoglobin $<90 \mathrm{~g} / \mathrm{L}$ (haemoglobin $<100 \mathrm{~g} / \mathrm{L}$ in infants $<4$ weeks)

- Platelets $<100 \times 10^{9} / \mathrm{L}$

Neutrophils $<1.0 \times 10^{9} / \mathrm{L}$

- Hypertriglyceridaemia and/or hypofibrinogenaemia

- Fasting triglycerides $\geq 3.0 \mathrm{mmol} / \mathrm{L}$ (ie, $\geq 265 \mathrm{mg} / \mathrm{dL}$ )

- Fibrinogen $\leq 1.5 \mathrm{~g} / \mathrm{L}$

- Hemophagocytosis in bone marrow or spleen or lymph nodes. No evidence of malignancy.

- Low or no NK cell activity (according to local laboratory reference)

- Ferritin $\geq 500 \mathrm{mg} / \mathrm{L}$

- sCD25 (ie, soluble IL-2 receptor) $\geq 2400 \mathrm{U} / \mathrm{mL}$

NK, natural killer; sCD25, soluble CD25.

basis, COVID-19 with pulmonary involvement could be considered itself a hyperferritinaemic syndrome, regardless of concomitant sHLH. ${ }^{12}$ The identification of hemophagocytosis features in COVID-19 lung tissue, the assessment of ferritin and proinflammatory cytokines trends overtime, along with receiver operating characteristic curves computed with data of patients with COVID19 would add significant knowledge on this matter. In particular, this would allow (1) to confirm whether COVID-19 is yet another hyperferritinemic syndrome, (2) to clarify whether current HScore cut-off values are reliable in these patients or should be redefined and (3) to understand the actual extent of the cytokine storm in all patients with COVID-19, regardless of the severity, and ultimately to support clinicians in the decision of when and in whom to start immunomodulating therapy and with which compound. Moreover, it is important to understand whether in severe forms of COVID-19associated pneumonia, ferritin is a mere acute phase protein or rather an active pathogenic mediator. In addition, in light of the pro-inflammatory role of ferritin enriched in heavy subunits (heavy ferritin), it could be of interest to evaluate the heavy:light ferritin ratio in COVID-19. ${ }^{18}$ Finally, the potential usefulness of the ferritin:erythrocyte sedimentation rate ratio, which may be an immediate, inexpensive and easy-to-implement biomarker, needs to be considered. ${ }^{19}$

\section{COVID-19 and cytokine storms in children}

Despite earlier reports outlined that COVID-19 morbidity and mortality in children was very low, ${ }^{20}$ very recently, 
several children with a novel multisystem inflammatory disease resembling toxic shock syndrome (TSS) and atypical Kawasaki disease (KD) with proven SARS-CoV2 infection have been observed in the UK. Likewise, an increased frequency of $\mathrm{KD}$ has been reported in Italy. ${ }^{21} 22$ TSS is a rare, life-threatening condition due in most cases to toxins produced by Staphylococcus aureus bacteria but also related to group A Streptococcus. TSS is characterised by fever, diffuse macular erythroderma, desquamation 1-2 weeks after onset of rash, hypotension and multisystem involvement of three or more of the following organ systems: gastrointestinal, muscular, mucous membrane, renal, hepatic, hematologic, central nervous system. ${ }^{23}$ However, to be classified as probable or definite TSS, the clinical picture is necessary but not sufficient and either of the two above-mentioned bacteria need to be isolated from biologic samples. In the current circumstances, whether viral isolates may be also considered remains to be elucidated, as it is tempting to speculate that the SARS-CoV2, alone or in combination, could be triggering or facilitating the TSS. On the other hand, KD is an acute systemic vasculitis characterised by fever, nonsuppurative conjunctival injection, rash, oral mucositis, extremity changes and cervical lymphadenopathy, and along with scarlet fever, meningococcaemia and other conditions represent a differential diagnosis of TSS. It comes with no surprise that elevated IL-1b and IL-6 levels in addition to a monotype profile shifted to intermediate monocytes are common hallmarks in $\mathrm{KD} .{ }^{24}$ MAS is a complication in about $2 \%$ of patients with $\mathrm{KD}$; however, the incidence is likely underestimated as many clinical and laboratory features of both diseases overlap and the HLH 2009 criteria have low sensitivity and specificity for the diagnosis of MAS complicating KD. ${ }^{25}$ Recently, classification criteria for MAS in the paediatric population have been released and will allow to provide more reliable epidemiological data. ${ }^{26}$ On this basis, it is conceivable that what is defined as 'a novel multisystem inflammatory disease' may be yet another condition caused by the COVID-19-induced cytokine storm. The existing evidence, although limited, that unlike adults children with COVID19 and a good prognosis display normal ferritin levels, underscores the importance to measure this molecule in those with severe COVID-19 and the above-mentioned multisystem inflammatory disease. ${ }^{27-29}$ Furthermore, comparing the vascular involvement in the young and lung involvement in the adult/elderly will help not only to characterise the pathogenic mechanisms behind SARSCoV2 infection but also to gain understanding towards differential responses across the lifespan. The latter may be useful to define personalised medicine algorithms or better adjust the existing ones.

\section{Therapeutic perspectives and concluding remarks}

Most of the aspects of the hyperinflammation during COVID-19 remain therefore to be elucidated, and it is not known who will develop the cytokine storm, when and at which extent. Nonetheless, several immunomodulating agents inhibiting the activity of IFN $\gamma$, IL- $1 \beta$, TNF $\alpha$, IL-6 and the Janus kinase 1 and 2-signal transducer and activator of transcription pathway are under investigation for the treatment of selected cases of COVID-19. Despite being the mainstay of treatment of hyperinflammation, immunomodulatory agents are double-edged swords in a viral infection. They must be managed with caution, and special attention needs to be considered when referring to non-viral hyperinflammation treatment guidelines. In this regard, the location, the timing and the viral phase deserve some considerations that should guide treatment with these compounds.

First, it must be noted that disease timing is associated with distinct organs involved. Although originated in the lungs, the 'viral response phase' leads to a 'host inflammatory response phase' that is responsible for the hyperinflammation and thus, cytokine storm..$^{30}$ The exact overlap between infection phase and organ involvement needs to be more precisely defined, but it is tempting to speculate that underlying immune and inflammatory mechanisms differ across these phases, which will have clear implications for the therapy. It is conceivable that local immune activation in the lungs precedes the general hyperinflammation observed at the systemic level. The multi-organ failure observed in the late stages of COVID-19 severe infection is aligned with this notion. Alternatively, it may be hypothesised that an uncontrolled replication in the lungs due to a poor innate or $\mathrm{CD}^{+}$-mediated viral clearance may facilitate viral spreading via the vasculature to the systemic compartment. The fact that SARS-CoV2 binds the ACE2, which is expressed both in lungs and the vascular endothelium, may support this idea. An in-depth characterisation of the draining lymph node microenvironment should be pursued to better delineate infection kinetics and, hence, disease stages.

Second, during the acute inflammatory response to infective agents, we observe a rapid (within $30 \mathrm{~min}$ ) increase of $\mathrm{TNF} \alpha$ and IL- $1 \beta$ levels and a subsequent rise of IL- 6 . The high levels of IL-6 last for longer, while TNF $\alpha$ and IL-1 $\beta$ levels rapidly decrease (within 24-48 hours) ${ }^{31}$ Therefore, clinical trials need to clarify the outcome of treating patients with immunosuppressants at different disease stages and with different extent of pulmonary involvement. In this regard, interim results of the French CORIMUNO-TOCI open-label randomised controlled trial have been recently announced. ${ }^{32}$ A total of 129 patients being hospitalised for COVID-19 moderate or severe pneumonia not requiring intensive care upon admission were enrolled in the study and randomised 1:1 to receive standard of care with or without tocilizumab. A significantly lower proportion of patients needed ventilation (non-invasive or mechanical) or died by day 14 in the tocilizumab group confirming that early intervention may be a major determinant to ensure adequate tackling of the cytokine storm.

Furthermore, treatment with glucocorticoids may be beneficial for patients who develop ARDS, especially in the light of cost, availability, knowledge of the drug and previous use in hyperinflammation. However, they have been 
linked to exacerbation of COVID-19-associated lung injury. ${ }^{33}$ Trials are needed to investigate the optimal dosage and timing of glucocorticoids for COVID-19 treatment.

Finally, as only some of the patients with COVID-19 are (yet) being treated with antiviral agents, clinicians should pay attention to viral load and find a balance between ablating the cytokine storm via the use of immunomodulating agents without significantly affecting the host defence against the virus, thereby preventing uncontrolled replication. In this regard, the ideal scenario would be to combine treatments for the cytokine storm with effective antiviral therapy, thus reinforcing the need for disease stratification in benefit of the treatment decision-making process.

In conclusion, although the cytokine storm we observe in COVID-19 is similar to that observed in other diseases, this scenario is more complex than initially thought due to the heterogeneity of hyperinflammation. In addition, the question remains on whether is it a general phenomenon or rather a reaction of the lung possibly in relation to a massive viral invasion secondary to a lack of antiviral response. Only a deeper understanding of the origin and the different phases of the cytokine storm in relation to the whole clinical picture and disease evolution will allow to draw a definitive conclusion on the nature and natural history of this process. A better characterisation of the host immune response across disease stages, systemic/lung phase transition and viral lung tropism is needed. The acquisition of such notions will allow to confirm whether COVID-19 can be considered a hyperferritinaemic syndrome by itself and to optimise the management of this condition based on disease stage stratification, viral load and cytokine signature to ultimately tailor the therapeutic approach and improve disease prognosis.

\section{Contributors AA, FC and JRC drafted, read and approved the final manuscript.}

Funding This article did not receive any specific funding. JRC was supported by Sara Borrell (CD19/00120) and PI (PI16/00113) programmes from Instituto de Salud Carlos III (Ministry of Science and Innovation, Spain).

Competing interests None declared.

Patient consent for publication Not required.

Provenance and peer review Not commissioned; externally peer reviewed.

Open access This is an open access article distributed in accordance with the Creative Commons Attribution Non Commercial (CC BY-NC 4.0) license, which permits others to distribute, remix, adapt, build upon this work non-commercially, and license their derivative works on different terms, provided the original work is properly cited, appropriate credit is given, any changes made indicated, and the use is non-commercial. See: http://creativecommons.org/licenses/by-nc/4.0/

ORCID iDs

Alessia Alunno http://orcid.org/0000-0003-1105-5640

Francesco Carubbi http://orcid.org/0000-0003-1958-5136

Javier Rodríguez-Carrio http://orcid.org/0000-0002-0011-5102

\section{REFERENCES}

1 Zhou F, Yu T, Du R, et al. Clinical course and risk factors for mortality of adult inpatients with COVID-19 in Wuhan, China: a retrospective cohort study. Lancet 2020;395:1054-62.

2 Huang C, Wang Y, Li X, et al. Clinical features of patients infected with 2019 novel coronavirus in Wuhan, China. Lancet 2020;395:497-506.

3 Chenyang L, Shasha L, Liu Y. Role of immunosuppressive therapy in rheumatic diseases concurrent with COVID-19. Ann Rheum Dis 2020.
4 Zhou Y, Fu B, Zheng X, et al. Aberrant pathogenic GM-CSF+ T cells and inflammatory CD14+CD16+ monocytes in severe pulmonary syndrome patients of a new coronavirus. bioRxiv 2020.

5 Henderson LA, Canna SW, Schulert GS, et al. On the alert for cytokine storm: immunopathology in COVID-19. Arthritis Rheumatol 2020.

6 Mehta P, McAuley DF, Brown M, et al. COVID-19: consider cytokine storm syndromes and immunosuppression. Lancet 2020;395:1033-4.

7 McGonagle D, Sharif K, O'Regan A, et al. The role of cytokines including interleukin-6 in COVID-19 induced pneumonia and macrophage activation syndrome-like disease. Autoimmun Rev 2020.

8 Murthy H, lqbal M, Chavez JC, et al. Cytokine release syndrome: current perspectives. Immunotargets Ther 2019;8:43-52.

9 Ramos-Casals M, Brito-Zerón P, López-Guillermo A, et al. Adult haemophagocytic syndrome. Lancet 2014;383:1503-16.

10 La Rosée P, Horne A, Hines M, et al. Recommendations for the management of hemophagocytic lymphohistiocytosis in adults. Blood 2019;133:2465-77.

11 Conti P, Ronconi G, Caraffa A, et al. Induction of pro-inflammatory cytokines (IL-1 and IL-6) and lung inflammation by COVID-19: anti-inflammatory strategies. J Biol Regul Homeost Agents 2020;34:2.

12 Nicholls JM, Poon LL, Lee KC, et al. Lung pathology of fatal severe acute respiratory syndrome. Lancet 2003;361:1773-8.

13 Henter JI, Horne A, Aricó M, et al. HLH-2004:diagnostic and therapeutic guidelines for hemophagocytic lymphohistiocytosis. Pediatr Blood Cancer 2007;124-31.

14 Fardet L, Galicier L, Lambotte O, et al. Development and validation of the HScore, a score for the diagnosis of reactive hemophagocytic syndrome. Arthritis Rheumatol 2014;66:2613-20.

15 Ruan Q, Yang K, Wang W, et al. Clinical predictors of mortality due to COVID-19 based on an analysis of data of 150 patients from Wuhan, China. Intensive Care Med 2020.

16 Rosário C, Zandman-Goddard G, Meyron-Holtz EG, et al. The hyperferritinemic syndrome: macrophage activation syndrome, Still's disease, septic shock and catastrophic antiphospholipid syndrome. BMC Med 2013;11:185.

17 Saper VE, Chen G, Deutsch GH, et al. Emergent high fatality lung disease in systemic juvenile arthritis. Ann Rheum Dis 2019;78:1722-31.

18 Recalcati S, Invernizzi P, Arosio P, et al. New functions for an iron storage protein: the role of ferritin in immunity and autoimmunity. J Autoimmun 2008;30:84-9.

19 Eloseily EMA, Minoia F, Crayne CB, et al. Ferritin to erythrocyte sedimentation rate ratio: simple measure to identify macrophage activation syndrome in systemic juvenile idiopathic arthritis. ACR Open Rheumatol 2019;1:345-9.

20 Sinha IP, Harwood R, Semple MG, et al. COVID-19 infection in children Lancet Respir Med 2020.

21 Available https://picsociety.uk/news/pics-statement-regarding-novelpresentation-of-multi-system-inflammatory-disease/

22 Available https://www.gaslini.org/comunicati-stampa/covid-19-neibambini-possibile-causa-del-notevole-incremento-dei-casi-dimalattia-di-kawasaki-riscontrati-nelle-ultime-settimane/

23 Available https://wwwn.cdc.gov/nndss/conditions/toxic-shocksyndrome-other-than-streptococcal/case-definition/2011/

24 Menikou S, Langford PR, Levin M. Kawasaki disease: the role of immune complexes revisited. Front Immunol 2019.

25 Wang W, Gong F, Zhu W, et al. Macrophage activation syndrome in Kawasaki disease: more common than we thought? Semin Arthritis Rheum 2015;44:405-10.

26 Ravelli A, Minoia F, Davì S, et al. Classification criteria for macrophage activation syndrome complicating systemic juvenile idiopathic arthritis: a European League Against Rheumatism/American College of Rheumatology/Paediatric Rheumatology International Trials Organisation Collaborative Initiative. Ann Rheum Dis 2016;2016:481-9.

$27 \mathrm{Su}$, MaX, Yu H, et al. The different clinical characteristics of corona virus disease cases between children and their families in China - the character of children with COVID-19. Emerg Microbes Infect 2020;9:707-13.

28 Molloy EJ, Bearer CF. COVID-19 in children and altered inflammatory responses. Pediatr Res 2020.

29 Choi SH, Kim HW, Kang JM, et al. Epidemiology and clinical features of coronavirus disease 2019 in children. Clin Exp Pediatr 2020;63:125-32.

30 Siddiqi HK, Mehra MR. COVID-19 illness in native and immunosuppressed states: a clinical-therapeutic staging proposal. $J$ Heart Lung Transplant 2020;39:405-7.

31 Schulte W, Bernhagen J, Bucala R. Cytokines in sepsis: potent immunoregulators and potential therapeutic targets: an updated view. Mediators Inflamm 2013;2013:1-16.

32 Available https://www.aphp.fr/contenu/tocilizumab-improvessignificantly-clinical-outcomes-patients-moderate-or-severe-covid-19

33 Russell CD, Millar JE, Baillie JK. Clinical evidence does not support corticosteroid treatment for 2019-nCoV lung injury. Lancet 2020;395:473-5. 